\title{
Diferencias en la Composición Corporal y Somatotipo de Escolares de Etnia Mapuche y no Mapuche de la Comuna de Temuco - Chile
}

\author{
Ethnic Differences in Body Composition and Somatotype in Mapuche \\ and Non-Mapuche School Children from Temuco-Chile
}

José Bruneau-Chávez*; Vanesa España-Romero**; Morín Lang-Tapia**** \& Palma Chillón Garzón *****

BRUNEAU-CHÁVEZ, J.; ESPAÑA-ROMERO, V.; LANG-TAPIA, M. \& CHILLÓN, G. P. Diferencias en la composición corporal y somatotipo de escolares de etnia Mapuche y no Mapuche de la comuna de Temuco-Chile. Int. J. Morphol., 33(3):988-995, 2015.

RESUMEN: Los cambios culturales y demográficos en la etnia Mapuche chilena, han generado modificaciones en estilos de vida y la estructura morfofuncional de su población, incentivando de esta forma el estudio del estado de salud en jóvenes chilenos. El objetivo de este estudio fue analizar las diferencias en composición corporal y somatotipo entre niños Mapuche y no-Mapuche de la Comuna de Temuco en Chile. Participaron 122 niños Mapuche y 146 niños no-Mapuche entre 10 y 13 años. Se recogieron medidas de peso, talla; pliegues cutáneos y diámetros óseos; siguiendo el protocolo de la Sociedad Internacional para el desarrollo de la cineantropometría (ISAK). Se determinó el índice de masa corporal (IMC), estado nutricional, grasa corporal total y somatotipo (endomorfía, mesomorfía y ectomorfía). Los resultados indican que los niños Mapuche de 10 años presentaron valores menores de IMC (p $\leq 0,01$ ), grasa corporal total $(\mathrm{p} \leq 0,001)$, así como de endomorfia y mesomorfia $(\mathrm{p} \leq 0,01)$ respecto a los no-Mapuche. Los niños Mapuche de 1112 años presentaron valores menores de grasa corporal total $(\mathrm{p} \leq 0,01)$ y de endomorfia y ectomorfia ( $\mathrm{p} \leq 0,05)$, comparados con los noMapuche. Los niños Mapuches de 13 años presentaron valores menores de grasa corporal total ( $\mathrm{p} \leq 0,001)$ y de endomorfia y ectomorfía $(\mathrm{p} \leq 0,01)$ que el no-Mapuche. Los escolares Mapuches tienen menor grasa corporal que los niños no-Mapuche, el somatotipo predominante en la etnia Mapuche es el mesomórfico, observándose un componente endomórfico menor cuanto mayor es la edad. Son necesarias políticas de salud pública en los jóvenes, fundamentalmente educativa, para garantizar niveles adecuados de calidad de vida en ambas poblaciones Mapuches y no-Mapuche.

PALABRAS CLAVE: Composición corporal; Somatotipo; Estado nutricional; Escolares; Etnia Mapuche.

\section{INTRODUCCIÓN}

La obesidad en países desarrollados se ha convertido en un problema de salud pública, no escapando de esta situación los jóvenes de Chile. La OMS, define la obesidad como una enfermedad crónica y multifactorial, y es el resultado de la unión de factores biológicos, genéticos y ambientales. Los factores más estudiados de la obesidad están relacionados con los estilos de vida, actividad física, nivel socioeconómico, sexo, y edad; por lo tanto es compleja y dinámica (Peña \& Bacallao, 2001). Asociado a este fenómeno, países desarrollados han presentado un especial interés en la investigación del comportamiento morfofuncional de sus habitantes, en relación a los niveles de aptitud física, estado nutricional, forma y composición corporal (Aliaga, 2011). El problema de la obesidad en Chile, afecta a una gran parte de la población (Ministerio de Salud, 2010), no quedando ajena a este problema la etnia Mapuche, pueblo originario de la zona sur de chile.

La etnia Mapuche está sufriendo una migración de lo rural a lo urbano, adaptándose a los cambios de la sociedad actual, desarrollando conductas y estilos de vida propios de las áreas urbanas e industrializadas donde predomina un ambiente obesogénico (Martínez et al., 2012). Hoy en día, cerca del 65\% del total de la población indígena de Chile, habita en las zonas urbanas (Del Popolo \& Oyarce, 2005). Por esta razón, es de interés analizar el impacto de estos cambios sociales en las características de composición corporal y somatotipo de los jóvenes de la etnia Mapuche.

\footnotetext{
* Pedagogía en Educación Física, Facultad de Educación, Universidad Autónoma de Chile, sede Temuco, Chile.

** Grupo de Investigación GALENO CTS-158, Departamento de Educación Física, Facultad de Ciencias de la Educación, Universidad de Cádiz, Cádiz, España.

**** Departamento de Ciencias de la Rehabilitación y del Movimiento Humano, Universidad de Antofagasta, Antofagasta, Chile.

**** Departamento de Educación Física y Deportiva, Universidad de Granada, Granada, España.
} 
En el contexto internacional, investigaciones previas han identificado diferencias en la composición corporal y el somatotipo entre diferentes etnias en Australia (Kagawa et al., 2009), Nueva Zelanda (Goulding et al., 2010) y Reino Unido (Nightingale et al., 2013). En Chile, la mayoría de los estudios centrados en obesidad se han realizado principalmente en población no-Mapuche (Liberona et al., 2011), sin embargo también existen estudios en pueblos étnicos originarios tales como Huilliches (Arcay, 1999) y Mapuches (Silva et al., 2008). En el caso particular de la etnia Mapuche del sector urbano y rural de la ciudad de Temuco, se reportan investigaciones de su somatotipo e índice nutricional, indicando que la mayoría de la población escolar y adolescente de ese sector presenta una clasificación somatotipica endomesomórfico tendiente a la obesidad en la población femenina y mesoendomórfico en la población masculina (Silva et al., 2008). No obstante, hasta donde los autores sabemos no se han analizado las diferencias entre la composición corporal y el somatotipo de la etnia Mapuche y no-Mapuche en Chile. Un análisis detallado de estos componentes ayudaría a establecer estrategias de intervención en materia de salud pública en una sociedad donde ambos conviven. Por lo tanto, el objetivo del presente estudio es determinar si existen diferencias en composición corporal y somatotipo entre niños Mapuche y no-Mapuche, pertenecientes a colegios municipales que habitan en la Comuna de Temuco de la IX Región de Chile.

\section{MATERIAL Y MÉTODO}

Estudio observacional de corte transversal, en el que se establecen dos grupos diferenciadores en base a la etnia Mapuche vs. la no-Mapuche. La población estuvo constituida por todos la población escolar perteneciente al segundo ciclo básico de los colegios municipales de la ciudad de Temuco, Chile ( $\mathrm{n}=5100)$, y los criterios de inclusión fueron: ser de sexo masculino, estar matriculado en alguno de los colegios municipales de la ciudad, encontrarse en el rango de los 10 y 13 años de edad, para los sujetos Mapuches poseer los dos apellidos. La muestra fue seleccionada mediante muestreo probabilístico simple, seleccionando al azar 15 niños de cada curso del segundo ciclo de enseñanza básica de cada una de los 8 colegios públicos seleccionados por sorteo, con lo que se obtuvo 122 niños de descendencia Mapuche procedentes de 4 colegios ubicados en zonas rurales, y 146 niños de descendencia no-Mapuche que procedían de 4 colegios de la ciudad de Temuco.

El presente estudio contó con el consentimiento informado de las familias de los niños y fue desarrollado siguiendo lo expuesto en la Declaración de Helsinki, respecto al trabajo con seres humanos.
La composición corporal se estimó a través de antropometría, basándose en las instrucciones de la Sociedad Internacional para el Desarrollo de la Cineantropometría (ISAK). Las medidas antropométricas fueron realizadas por un equipo de cuatro profesores de Educación Física, especialistas en evaluación antropométrica, todos ellos en posesión de la certificación ISAK nivel II en antropometría, y tuvieron lugar en los centros educativos.

El peso corporal y la talla, se midió con el participante en ropa interior y descalzo, registrándose el peso en una báscula (SECA modelo 220, Alemania) con precisión de $0,1 \mathrm{~kg}$ y la talla, en un tallimetro con precisión de $0,1 \mathrm{~cm}$, modelo SECA.

Los pliegues cutáneos valorados en este estudio fueron tricipital, subescapular, supraespinal y pantorrilla. Se tomaron utilizando un plicómetro marca (Harpenden modelo Baty International AH15 9LR. UK) con una presión constante de $10 \mathrm{~g} / \mathrm{mm}^{2}$ de superficie de contacto. Los perímetros corporales que se evaluaron fueron brazo relajado, brazo contraído, cintura y pantorrilla, utilizando cintas metálicas inextensibles de 0,5 cm marca Lufkin WP-606 (rango 0$150 \mathrm{~cm}$ ). Los diámetros óseos evaluados fueron fémur y húmero, utilizando calibres Campbell 10 (Rosscraft SRL, Argentina).

Tras las mediciones antropométricas, se realizaron cálculos para definir: a) índice de masa corporal [IMC $=$ (peso corporal $(\mathrm{kg}) /$ talla $\left.(\mathrm{m})^{2}\right)$ ]; b) estado nutricional, clasificando a los niños según su IMC en: bajo peso (IMC menor o igual a $14,8 \mathrm{~kg} / \mathrm{m}^{2}$ ), normal (IMC entre $14,9 \mathrm{~km} / \mathrm{m}^{2}$ y 20,5 $\mathrm{kg} / \mathrm{m}^{2}$ ), sobrepeso (IMC entre 20,6 y $23,6 \mathrm{~kg} / \mathrm{m}^{2}$ ) y obesidad (IMC mayor de $23,7 \mathrm{~kg} / \mathrm{m}^{2}$ ) (Amigo et al., 2008), c) grasa corporal total (GCT), en base a las variables de masa grasa y porcentaje de grasa corporal mediante el método propuesto por Slaugther (1988), e d) indicador de sobrepeso y obesidad de acuerdo al porcentaje de GCT $>20 \%$ (Hoyo \& Sañudo, 2007).

El cálculo de somatotipo se realizó con las ecuaciones propuestas por Carter \& Heath (1990). Además, a través de la Distancia de Dispersión del Somatotipo (DDS), calculada por método Hebbelinck (Hebbelinck et al., 1975), se determinó la distancia entre somatotipos de Mapuche vs. noMapuche en la somatocarta (distancia entre dos somatopuntos). Un valor de DDS mayor de 2 indica que hay diferencias significativas entre ambos grupos.

Análisis estadístico. Se utilizó planilla Microsoft Excel versión 7.0 para el vaciado preliminar de datos y para el tratamiento estadístico se usó el programa computacional SPSS versión 20.0 (SPSS Inc. Chicago) fijando el mínimo de sig- 
nificación en el 5\%. Los valores se presentan como Media \pm Desviación Típica en variables continuas y como frecuencias en variables categóricas. La normalidad de la distribución de las variables se estudió mediante el test de Kolmogorov-Smirnov. Se encontraron efectos de interacción para la edad en relación con las variables de composición corporal y por tanto los resultados se presentan para niños de 10, 11-12 y 13 años, respectivamente. En variables continuas (IMC y grasa corporal) se realizó análisis de varianza de 1 vía (ANOVA) utilizando la variable etnia (Mapuche vs. no-Mapuche) como factor fijo en cada grupo de edad establecido (es decir, en los grupos de 10 años, 11-12 años y 13 años). En variables categóricas (estado nutricional, somatotipo $\mathrm{y}$ el indicador de sobrepeso y obesidad de acuerdo al porcentaje de GCT $\geq 0 \%$ ) se realizó chi-cuadrado para analizar diferencias en las proporciones de la variable objeto.

\section{RESULTADOS}

Tabla I. Características descriptivas de parámetros de composición corporal en Mapuche y no-Mapuche de 10 años de edad.

\begin{tabular}{|c|c|c|c|c|}
\hline & & $\begin{array}{c}\text { Total } \\
(n=44)\end{array}$ & $\begin{array}{c}\text { No-mapuche } \\
(n=24)\end{array}$ & $\begin{array}{c}\text { Mapuche } \\
(n=20)\end{array}$ \\
\hline $\operatorname{IMC}\left(\mathrm{kg} / \mathrm{m}^{2}\right)$ & & $18,3(1,2)$ & $18,8(1,1)$ & $17,8(1,2)$ \\
\hline \multirow{4}{*}{$\begin{array}{l}\text { Estado Nutricional } 1 \\
(\%)\end{array}$} & Bajo peso & 0 & 0 & 0 \\
\hline & Normal & 77,9 & 70,8 & $85 \mathrm{c}$ \\
\hline & Sobrepeso & 22,1 & 29,2 & $15 \mathrm{c}$ \\
\hline & Obesidad & 0 & 0 & 0 \\
\hline \multirow[t]{4}{*}{ Grasa corporal total } & Masa grasa 2(kg) & $7(1,4)$ & $8,2(2,1)$ & $5,7(0,7)^{\mathrm{c}}$ \\
\hline & Masa grasa $3(\mathrm{~kg})$ & $4,5(1)$ & $5,4(1,2)$ & $3,6(0,7)^{\mathrm{c}}$ \\
\hline & Porcentaje de gra sa corporal ${ }^{2}(\%)$ & 20,5 & 22,8 & $18,2^{\mathrm{c}}$ \\
\hline & Porcentaje de gra sa corporal ${ }^{2}(\% \geq 20 \%)$ & 47,5 & 75,0 & $20,0 \mathrm{c}$ \\
\hline
\end{tabular}

Los valores son mostrados como media (desviación típica) a menos que otra información sea mostrada en la tabla; $1=$ Puntos de corte de IMC según Instituto de tecnología de los alimentos de la Universidad de Chile, INTA. Masa grasa 2= Calculado según Slaughter (tríceps y subescapular); $3=$ Calculado según Slaughter (tríceps y gemelos). $b=p \leq 0,01 ; c=p \leq 0,001$.

Tabla II. Características descriptivas de parámetros de composición corporal en Mapuche y no-Mapuche de 11-12 años de edad.

\begin{tabular}{|c|c|c|c|c|}
\hline & & $\begin{array}{c}\text { Total } \\
(n=152)\end{array}$ & $\begin{array}{l}\text { No-mapuche } \\
\quad(n=82)\end{array}$ & $\begin{array}{c}\text { Mapuche } \\
(n=70)\end{array}$ \\
\hline $\mathrm{IMC}\left(\mathrm{kg} / \mathrm{m}^{2}\right)$ & & $19,4(2,4)$ & $19,1(2,1)$ & $19,6(2,7)$ \\
\hline \multirow{4}{*}{$\begin{array}{l}\text { Estado Nutricional I } \\
(\%)\end{array}$} & Bajo peso & 2,03 & 1,2 & $2,85^{*}$ \\
\hline & Normal & 73,82 & 71,95 & $75,7^{\mathrm{b}}$ \\
\hline & Sobrepeso & 19,45 & 23,2 & $15,7^{\mathrm{c}}$ \\
\hline & Obesidad & 4,7 & 3,65 & $5,75^{*}$ \\
\hline \multirow[t]{4}{*}{ Grasa corporal total } & Masa grasa $2(\mathrm{~kg})$ & $7,3(2,4)$ & $7,3(2,6)$ & $7,2(2,1)^{b}$ \\
\hline & Masa grasa $3(\mathrm{~kg})$ & $6,0(1,7)$ & $6,7(1,9)$ & $5,2(1,5)$ \\
\hline & Porcentaje de grasa corporal ${ }^{2}(\%)$ & 20,6 & 22,8 & $18,5 \mathrm{c}$ \\
\hline & Porcentaje de grasa corporal ${ }^{2}(\% \geq 20 \%)$ & 34,35 & 48,7 & $20,0 \mathrm{c}$ \\
\hline
\end{tabular}

Los valores son mostrados como media (desviación típica) a menos que otra información sea mostrada en la tabla; $1=$ Puntos de corte de IMC según Instituto de tecnología de los alimentos de la Universidad de Chile, INTA (REFERENCIA) Masa grasa 2= Calculado según Slaughter (tríceps y subescapular); $3=$ Calculado según Slaughter (tríceps y gemelos).

$\mathrm{a}=\mathrm{p} \leq 0,05 ; \mathrm{b}=\mathrm{p} \leq 0,01 ; \mathrm{c}=\mathrm{p} \leq 0,001 ; *$ Prueba de chi-cuadrado no se realizo por baja muestra. 
BRUNEAU-CHÁVEZ, J.; ESPAÑA-ROMERO, V.; LANG-TAPIA, M. \& CHILLÓN, G. P. Diferencias en la composición corporal y somatotipo de escolares de etnia Mapuche y no Mapuche de la comuna de Temuco-Chile. Int. J. Morphol., 33(3):988-995, 2015.

Tabla III. Características descriptivas de parámetros de composición corporal en Mapuche y no-Mapuche de 13 años de edad.

\begin{tabular}{|c|c|c|c|c|}
\hline & & $\begin{array}{c}\text { Total } \\
(n=72)\end{array}$ & $\begin{array}{c}\text { No-mapuche } \\
(n=40)\end{array}$ & $\begin{array}{c}\text { Mapuche } \\
(n=32)\end{array}$ \\
\hline $\operatorname{IMC}\left(\mathrm{kg} / \mathrm{m}^{2}\right)$ & & $21(2,3)$ & $21,5(2,7)$ & $20,6(1,8)$ \\
\hline \multirow{4}{*}{$\begin{array}{l}\text { Estado Nutricional } 1 \\
(\%)\end{array}$} & Bajo peso & 0 & 0 & 0 \\
\hline & Normal & 60,3 & 42,5 & $78,1^{\mathrm{c}}$ \\
\hline & Sobrepeso & 39,7 & 57,5 & $21,9^{\mathrm{c}}$ \\
\hline & Obesidad & 0 & 0 & 0 \\
\hline \multirow[t]{4}{*}{ Grasa corporal total } & Masa grasa ${ }^{2}(\mathrm{~kg})$ & $8,9(2,4)$ & $11,1(3,3)$ & $6,6(1,4)^{\mathrm{c}}$ \\
\hline & Masa grasa ${ }^{3}(\mathrm{~kg})$ & $6,5(1,8)$ & $7,5(2,5)$ & $5,4(1,1)^{\mathrm{c}}$ \\
\hline & Porc entaje de grasa corpora $12(\%)$ & 17,2 & 19,9 & $14,4^{\mathrm{c}}$ \\
\hline & Porc entaje de grasa corporal $2(\% \geq 20 \%)$ & 32,1 & 55,0 & $9,3 \mathrm{c}$ \\
\hline
\end{tabular}

Los valores son mostrados como media (desviación típica) a menos que otra información sea mostrada en la tabla; $1=$ Puntos de corte de IMC según Instituto de tecnología de los alimentos de la Universidad de Chile, INTA (REFERENCIA) 2= Calculado según Slaughter (tríceps y subescapular); $3=$ Calculado según Slaughter (tríceps y gemelos). a $=\mathrm{p} \leq 0,05 ; \mathrm{b}=\mathrm{p} \leq 0,01 ; \mathrm{c}=\mathrm{p} \leq 0,001$.

Los datos de los componentes del Somatotipo atendiendo a las diferencias entre Mapuche y no-Mapuche para la edad de 10 años, 11-12 años y 13 años se presentan en las Figuras 1,2 y 3 ,respectivamente. En el grupo de edad de 10 años se observan diferencias significativas entre ambos grupos para los valores específicos de endomorfia y mesomorfia a favor del no-Mapuche $(\mathrm{p} \leq 0,01)$ y ectomorfía a favor del Mapuche $(p \leq 0,01)$. El somatotipo clasifica tanto a los niños Mapuches como al no-Mapuche como endomesomórfico. En cuanto a la DDS, esta logra un valor de 2,23, indicando que existen diferencias significativas entre los somatotipos de los niños Mapuche y no-Mapuche (Fig. 1).

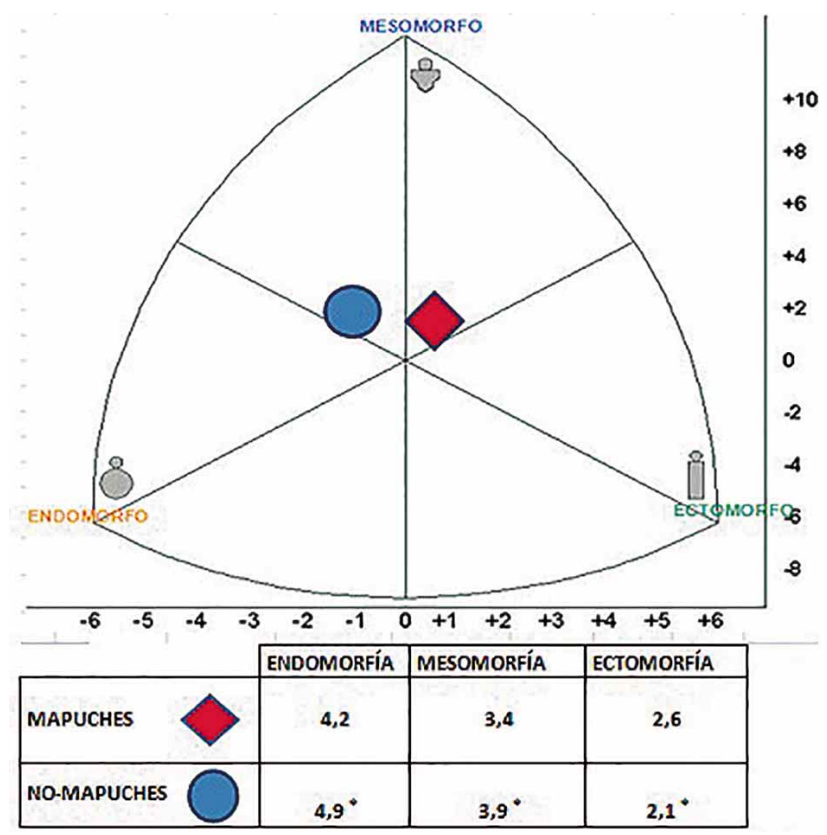

Fig. 1. Somatotipo en niños Mapuches y no-Mapuches de 10 años calculado según Carter \& Heath (1990). Distancia de Dispersión Somatotípica (DDS) calculada según Hebbelinck et al. (1975). *= $\mathrm{p} \leq 0,01 ; \mathrm{DDS}=2,23$.
En el grupo de edad de 11-12 años, se observan diferencias significativas entre ambas etnias para los valores específicos de endomorfia y ectomorfia a favor del noMapuche $(p \leq 0,05)$ y mesomorfia a favor del Mapuche $(\mathrm{p} \leq 0,05)$. El somatotipo clasifica al grupo Mapuche como mesoendomórfico y al grupo no-Mapuche como endomesomorfo. En cuanto a la DDS, esta logra un valor de 1,91 , indicando que no existen diferencias significativas entre los somatotipos de niños Mapuche y no-Mapuche (Fig. 2).

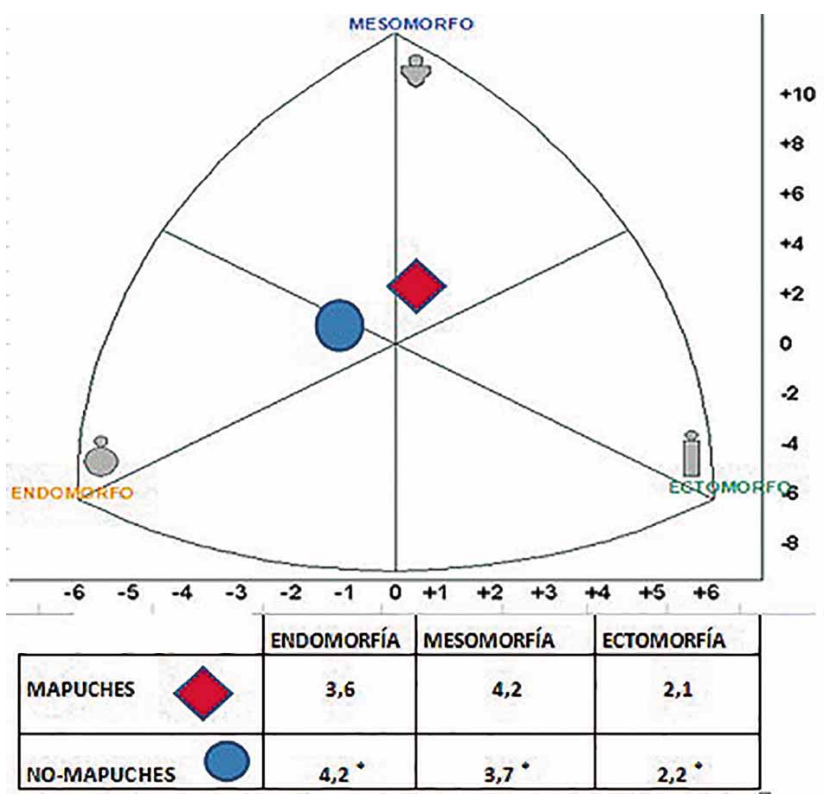

Fig. 2. Somatotipo en niños Mapuches y no-Mapuches de 1112años calculado según Carter \& Heath (1990). Distancia de Dispersión Somatotípica (DDS) calculado según Hebbelinck et al. $(1975) . *=p \leq 0,05 ;$ DDS $=1,91$.

En el grupo de edad de 13 años, se observan diferencias significativas entre ambas etnias para los valores específicos de endomorfia y ectomorfia a favor del no-Mapuche 
$(\mathrm{p} \leq 0,01)$ y mesomorfia a favor del Mapuche $(\mathrm{p} \leq 0,01)$. El somatotipo clasifica al grupo Mapuche como mesoendomórfico y al no-Mapuche como endomesomórfico. En cuanto a la DDS, este logra un valor de 2,43, indicando que existen diferencias significativas entre los somatotipos de niños Mapuche y no-Mapuche (Fig. 3).

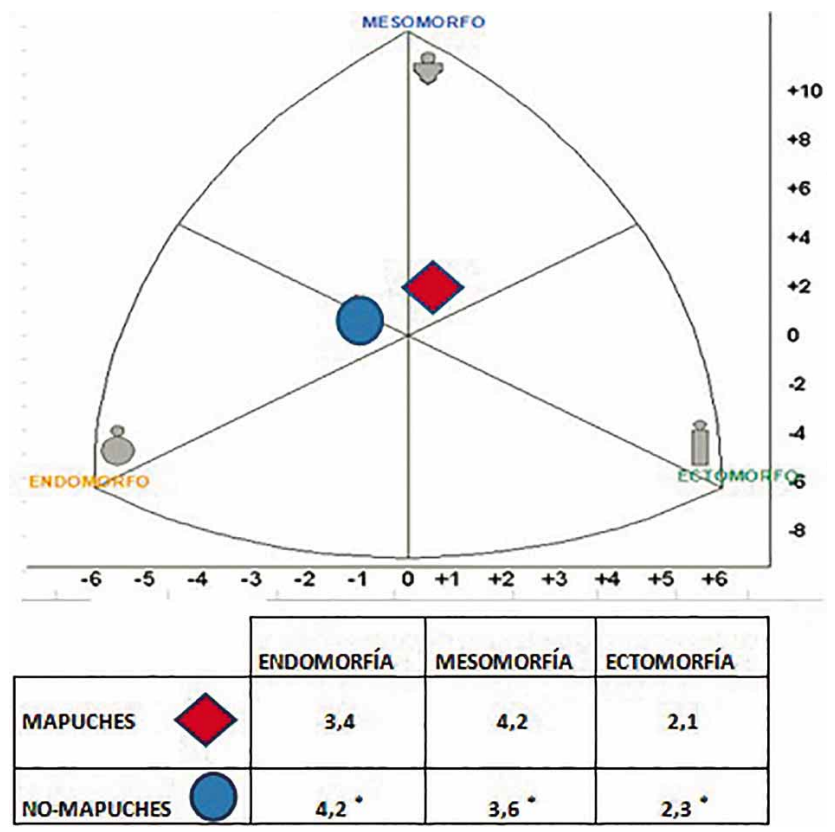

Fig. 3. Somatotipo en niños Mapuches y no-Mapuches de 13 años calculada según Carter et al., (1990). Distancia de Dispersión Somatotípica (DDS) calculado según Hebbelinck et al. (1975). *= $\mathrm{p} \leq 0,01 ; \mathrm{DDS}=2,43$.

\section{DISCUSIÓN}

En el presente estudio se ha analizado si existen diferencias en composición corporal y somatotipo en 122 niños de descendencia Mapuche y 146 niños de descendencia no-Mapuche con edades comprendidas entre 10 y 13 años que habitan en la Comuna de Temuco de la IX Región de Chile. Entre los resultados se puede observar que: i) no existen diferencias estadísticamente significativas entre Mapuches y no-Mapuches en la variable de IMC, excepto en el grupo de niños de 10 años donde los Mapuches presentan menores valores que los no-Mapuches; ii) existen diferencias significativas entre ambos grupos en el estado nutricional, observándose mayor porcentaje de Mapuches con normopeso y de no-Mapuches con sobrepeso; iii) los niños Mapuches presentan menos grasa corporal total respecto a los no-Mapuche en los tres grupos de edades estudiados; iv) el somatotipo predominante en la etnia Mapuche es el mesoendomórfico, en comparación con los no-Mapuche que tienen un componente endomesomórfico, además se observa en la población Mapuche menores valores en el componente endomórfico cuanto mayor es la edad del niño.

Las diferencias en IMC entre niños Mapuches y noMapuches no parecen haber sido estudiadas previamente, por lo tanto, nuestros resultados no pueden ser comparados con los de otros estudios realizados en esta línea. Sin embargo, hemos encontrado en la literatura artículos que comparan el ICM entre otras etnias chilenas. Por ejemplo, Espinoza-Navarro et al. (2009) compararon el IMC de niños chilenos Aymará y no-Aymará de 10 a 12 años que viven en zonas localizadas en diferentes altitudes, los del poblado de Putre (3.500 metros sobre el nivel del mar -msnm) y los del poblado de Azapa (500 msnm). Entre los resultados de ese estudio se observó que los niños no-Aymará (22 $\mathrm{kg} / \mathrm{m}^{2}$ en Putre y $23,4 \mathrm{~kg} / \mathrm{m}^{2}$ en Azapa) presentaron mayores valores de IMC que los niños Aymará ( 17 y $23 \mathrm{~kg} / \mathrm{m}^{2}$ en Putre y Azapa, respectivamente) independientemente de si vivían en altura o en la planicie, es decir, en Putre o Azapa. Similares resultados se observan en nuestro estudio en el grupo de niños no-Mapuches de 10 años ya que también presentaron mayores valores en la variable de IMC en comparación con el grupo de niños Mapuches de la misma edad. Sin embargo, los autores de este estudio no encontraron diferencias significativas en valores de IMC en el grupo de edades estudiadas. Las discrepancias observadas en IMC en el grupo de edad de 11 y 12 años podrían ser debidas a que son diferentes etnias las estudiadas, además de que entre Aymarás y no-Aymarás, la altitud podría ser un factor determinante del menor IMC encontrado en los niños que viven en altura. Por otro lado, es posible observar que los niños Mapuches de este estudio presentan valores de IMC mayores que los niños aymará que habitan en Putre, y menores que los del grupo aymará que viven en Azapa. También se compararon los valores de IMC de la etnia Mapuche con niños Huilliches, habitantes aborígenes del sur de Chile de la X Región, de 7 a 14 años obteniéndose valores de IMC menores en niños Mapuches $\left(19,4 \mathrm{~kg} / \mathrm{m}^{2}\right)$ que en niños Huilliches $\left(21 \mathrm{~kg} / \mathrm{m}^{2}\right)$.

Además de los estudios realizados en etnias chilenas, se observan en la literatura trabajos que comparan el IMC de niños australianos caucásicos con australianos aborígenes observándose diferentes resultados. Por ejemplo, Kagawa et al., estudiaron el IMC de 44 niños australianos caucásicos $\left(20,1 \mathrm{~kg} / \mathrm{m}^{2}\right)$, y el de 36 niños australianos aborígenes $\left(20,9 \mathrm{~kg} / \mathrm{m}^{2}\right)$ de 9 a 15 años, no encontrando diferencias significativas en IMC entre etnias. Sin embargo, se observa en otros trabajos que la etnia aborigen presenta menores valores en IMC que los no-aborígenes (Nightingale $e t$ al.; Aguilar et al., 2011). De forma general y siguiendo con los resultados observados en esos estudios, parece que los 
niños de etnias indígenas, Mapuches en este estudio, presentan valores menores de IMC que los no indígenas, noMapuches en este estudio. En el caso de nuestra muestra estudiada, estas diferencias podrían ser debidas a características genéticas, una alimentación más sana y equilibrada (pobre en grasas y rica en proteínas y carbohidratos), y un mayor nivel de actividad física en su rutina diaria. Un factor determinante en estas diferencias podría ser el nivel socioeconómico que es menor en los indígenas (Candia, 2006), posibilitando un menor acceso a alimentos procesados y utilizando la actividad física como medio básico de locomoción diaria.

Respecto al estado nutricional, no existen estudios precedentes que comparen niños Mapuches y no-Mapuches, pero sí existen estudios nacionales realizados sólo en la etnia Mapuche. Al comparar nuestros resultados, de acuerdo a las diferentes categorías nutricionales, hemos encontrado algunas diferencias en el grupo Mapuche de 10 a 13 años. Por ejemplo, Martínez et al. (2013), observaron en niños Mapuches de 9 a 12 años los porcentajes de $41,5 \%$ en normopeso, $35,4 \%$ en sobrepeso y $23,2 \%$ en obesidad. En tanto, otro estudio de Martínez et al. (2012), mostró porcentajes de $61,7 \%$ en normopeso, $20,1 \%$ en sobrepeso y $11,7 \%$ en obesidad en 154 niños Mapuches de 10 a 14 años. En ambos estudios, los porcentajes que expone Martínez et al. (2012, 2013), son menores a los encontrados en nuestro estudio en el grupo Mapuche para los clasificados en normopeso $(79,6 \%)$, y mayores para los clasificados en sobrepeso $(17,5 \%)$ y obesidad $(3,2 \%)$. Los bajos valores en la categoría obesidad respecto a la literatura previa, se podrían deber al nivel socioeconómico de la población y a la ubicación geográfica (rural y urbana) de los colegios. En los estudios de Martínez et al. $(2012,2013)$ y Silva et al. (2003), la población Mapuche es urbana y en el presente estudio la población Mapuche se ubica en zonas rurales de la comuna de Temuco. Estudios previos en jóvenes han encontrado mayores niveles de condición física en los jóvenes rurales respecto a los urbanos debido a diferente estilos de vida (Chillón et al., 2011).

Atendiendo a la grasa corporal total, los resultados evidencian que los niños Mapuches tienen menor masa grasa y menor porcentaje de grasa corporal que los niños noMapuches de su misma edad, incluso utilizando diferentes pliegues cutáneos para el cálculo de la ecuación de masa grasa (tríceps y subescapular vs. tríceps y pantorrilla). El porcentaje de masa grasa general obtenido atendiendo a los tres grupos de edad es de 17\% en el grupo Mapuche y 21,8\% en el grupo no-Mapuche, observándose que la media del porcentaje de grasa del grupo Mapuche es inferior al 20\% y el del grupo no-Mapuche es superior al 20\%, lo cual es un indicador de índices de sobrepeso u obesidad (Hoyo \& Sañudo).
En la literatura científica sólo hemos encontrado un artículo que analiza el porcentaje de grasa corporal en niños escolares que pertenecen a la etnia chilena Huilliche (Arcay). Arcay comparó los valores de porcentaje graso entre géneros en una muestra de 116 niños Huilliches de 7 a 14 años con $22,7 \%$ de grasa corporal, con 117 niñas Huilliches de 7 a 14 años con $28,6 \%$ de grasa corporal. Si comparamos los valores de porcentaje de grasa corporal de los niños Huilliches con los niños Mapuches de este estudio, se observan valores inferiores en los escolares Mapuches para los tres grupos etarios. Existen dos estudios realizados en población chilena que, sin incluir etniasaborígenes, sí analizan los valores de grasa corporal, utilizando la ecuación de Slaughter et al. (Lizana Arce et al., 2011; Silva et al., 2008). Lizana Arce et al., estudiaron 161 niños y niñas chilenos entre 10 y 18 años de Valparaíso en la V Región y observaron valores de $24,7 \%$ de grasa corporal. Silva et al. (2008), estudiaron a 50 escolares entre 14 y 16 años de Temuco en la IX - región, presentando valores de un $30 \%$ para los jóvenes varones. Se observa que en estos estudios en población escolar no aborigen, los valores de porcentaje de grasa corporal son mayores a los observados en el grupo no-Mapuche, siendo esta diferencia aún mayor en el grupo Mapuche.

En cuanto al somatotipo, los valores encontrados en este estudio indican que los niños de la etnia Mapuche presentaron valores mayores en el componente mesomórfico y valores menores en el componente endomórfico comparado con el no-Mapuche. En consonancia con nuestros resultados, existen diferentes estudios en Chile que han analizado el somatotipo en etnias Mapuches (Martínez et al., 2012, 2013; Silva et al., 2003) y Huilliches (Arcay). Martínez et al. (2013), en una población 122 niños y niñas de 9 a 12 años, obtuvo resultados muy similares a los nuestros, es decir, que la mesomorfía fue significativamente mayor en los escolares Mapuches y la endomorfía en los no-Mapuches. Otro estudio realizado por Silva et al. (2003) sobre somatotipo e índice de masa corporal en una muestra de adolescentes de ambos sexos de la ciudad de Temuco (Chile) en 208 niños Mapuches de 10 a 13 años de ambos sexos, muestra que los niños tienen una clasificación mesoendomorfa y las niñas endomesomorfa, aportando resultados similares en relación a la población de niños Mapuches. Además, Arcay, encontró diferencias entre sexo en niños Huilliches, clasificando los varones como mesoendomorfo, de tal forma que la clasificación somatotípica de los varones Huilliches es similar a la del grupo Mapuche de este estudio.

Por tanto, en concordancia con nuestro trabajo, los jóvenes Mapuches de los estudios de Silva et al. $(2003,2008)$ y Martínez et al. (2012, 2013), así como los escolares de la etnia Huilliche del estudio de Arcay, comparten la misma clasificación somatotipica (Mesoendomorfos). Esta infor- 
mación, resulta de especial interés ya que indica que la etnias estudiadas en Chile podrían presentar la misma clasificación somatotípica. De tal modo se puede inferir que en estos grupos podría existir similitud en cuanto a un mismo patrón de desarrollo músculo esquelético y una mayor presencia relativa de grasas en el cuerpo, debido quizás a factores similares de carga genética y de contexto geográfico y cultural.

A nivel internacional, los estudios han encontrado resultados controversiales en la diferencia en el somatotipo entre etnias y grupos (Kagawa et al.; Marrodán et al., 2001), si bien estudian otras etnias diferentes a los Mapuches. Kagawa et al., observó diferencias al comparar niños Australianos caucásicos con australianos aborígenes, resultando ser clasificados los caucásicos como mesoendomorfo y los aborígenes como endomesomorfo. En Latinoamérica, un estudio realizado por Marrodán et al., demostró diferencias es somatotipo al comparar niños y jóvenes mexicanos de 4 a 20 años de edad, de diversas procedencias. Los niños de Distrito Federal y Lomas de la Estancia promedialmente se clasificaron como mesoendomorfo, mientras que los habitantes en Chontales se clasificaron como mesoectomorfos. Las comparaciones entre etnias originales de diferentes países merecen una cautela especial, debido a la diferente carga genética y al variado contexto geográfico en el que se desarrollan, además de la diferente aplicación de políticas sociales aplicadas en cada país que incentivan o no de diferente forma a las culturas indígenas en temas sociales, de salud y educación fundamentalmente.

En nuestro estudio existen ciertas limitaciones que necesitan ser reconocidas. La muestra es únicamente masculina, por la dificultad de poder realizar medidas antropométricas al sexo femenino. Además, no se han considerado variables de índole socioeconómica, que podrían explicar en parte las diferencias observadas entre la etnia Mapuche y no-Mapuche. Sin embargo, el número de escolares incluidos y las variables antropométricas minuciosamente obtenidas son puntos fuertes que permiten conocer más profundamente las diferencias entre etnia Mapuche y no-Mapuche. Además, este es el primer estudio -bajo nuestro conocimiento- que presenta valores comparativos basados en estadística inferencial entre ambas etnias, Mapuches y no-Mapuches.

\section{CONCLUSIÓN}

Los resultados de nuestro estudio parecen indicar que los niños Mapuches tienen menos grasa corporal total y menos obesidad que los no-Mapuche. Además, los niños de etnia Mapuche presentan un somatotipo predominante mesoendomorfo mientras que los no-Mapuche endomesomórfico. Por lo tanto, desde el ámbito de la salud, parece necesario incluir estrategias educativas dirigidas fundamentalmente a la población joven no-Mapuche y a sus familiares y tutores, para reducir el exceso de grasa corporal, a través de iniciativas que fomenten una adecuada alimentación y un hábito de actividad física diario.

AGRADECIMIENTOS. Se agradece a los niños participantes del presente estudio, como a los centros educacionales que nos abrieron sus puertas permitiendo desarrollar las evaluaciones y registrar los datos utilizados.

BRUNEAU-CHÁVEZ, J.; ESPAÑA-ROMERO, V.; LANG-TAPIA, M. \& CHILLÓN, G. P. Diferencias en la composición corporal y somatotipo de escolares de etnia Mapuche y no Mapuche de la comuna de Temuco-Chile. Int. J. Morphol., 33(3):988-995, 2015.

SUMMARY: The cultural and demographic development of modern society has also affected the Mapuche ethnia and has influenced changes in lifestyles and in the morphofunctional structure of the population, particularly in young people. The purpose of this study was to determine differences in body composition and somatotype between Mapuche and non-Mapuche children from Temuco, Chile. A total of 122 Mapuche and 146 non-Mapuche children between 10 and 13 years old participated in the study. The measurements included were weight, height, skinfold thickness of triceps, subscapularis, supraspinatus and calf; perimeters of contracted and relaxed arm, waist and calf circumference; and humeral and femoral diameters, all following the procedures reference manual (ISAK). Several indexes were calculated: Body Mass Index (BMI), weight status (underweight, normoweight, overweight, obesity), total body fat (fat mass, percentage of fat mass and percentage of fat mass $>20 \%$ of obesity) and finally, somatotype (endomorph, ectomorph, mesomorph). Ten year-old Mapuche children had lower values for BMI ( $\mathrm{p} \leq 0,01)$, total body fat $(\mathrm{p} \leq 0.001)$, and endomorphic and mesomorphic values $(\mathrm{p} \leq 0.01)$, compared to non Mapuche children. Mapuche children of 11-12 years of age had lower values for total body fat ( $\mathrm{p} \leq 0.01$ ), and endomorphic and ectomorphic values $(\mathrm{p} \leq 0.05)$, compared to non-Mapuche children. Thirteen year-old Mapuche children had lower values for total body fat $(\mathrm{p} \leq 0.001)$, and endomorphic and ectomorphic values $(\mathrm{p} \leq 0.01)$, compared to non-Mapuche children. Mapuche children had lower fat mass than non-Mapuche children, and the main somatotype among Mapuche children is mesomorphic with a reduction of the endomorphic component with increasing age. Public policy for youth is required, mainly educational, to guarantee appropriate levels of quality of life for both Mapuche and non-Mapuche populations.

KEY WORDS: Body composition; Somatotype; Scholars; Young people; Mapuche. 


\section{REFERENCIAS BIBLIOGRÁFICAS}

Aguilar, A. C.; Pradilla, A.; Mosquera, M.; Gracia, A. B.; Ortega, J. G.; Leiva, J. H. \& Ramírez-Vélez R. Percentile values for physical condition for Cali, Colombian children and adolescents. Biomedica, 31(2): 242-9, 2011.

Aliaga, S. P. I. El rol del Estado en el deporte en Chile y en el derecho comparado. Tesis de Licenciado en Ciencias Jurídicas y Sociales. Santiago, Universidad de Chile, 2011. Disponible en: http:// tesis.uchile.cl/handle/2250/111100

Amigo, H.; Erazo, M.; Bustos, P.; Aguilar, C. \& Taibo, M. Vigilancia nutricional en escolares chilenos: Validez de la información. Rev. Med. Chile, 136(8):989-95, 2008

Arcay, M. R. Composición corporal en escolares de ascendencia indígena del norte, centro y sur de Chile. Educ. Fis. Chile, (248):26-30, 1999.

Candia, A. CASEN 2006: Análisis de los resultados preliminares y desafios pendientes. Santiago, Ministerio de Desarrollo Social, Gobierno de Chile, 2006. pp.43.

Carter, J. E. L. \& Heath, B. H. Somatotyping: Development and Applications. Cambridge, Cambridge University Press, 1990.

Chillón, P.; Ortega, F. B.; Ferrando, J. A. \& Casajus, J. A. Physical fitness in rural and urban children and adolescents from Spain. J. Sci. Med. Sport, 14(5):417-23, 2011.

Del Popolo, F. \& Oyarce, A. M. Población indigena de América Latina; perfil socidemográfico $n$ el marco dela CIPD y de las Metas del Milenio. Santiago de Chile, CEPAL. 2005. Disponible en: http:// www.cepal.org/celade/noticias/paginas/7/21237/delpopolo.pdf

Espinoza-Navarro, O.; Vega, C.; Urrutia, A.; Moreno, A. \& Rodríguez, H. Anthropometric Patterns and Oxygen Consumption (VO2) of Schoolchildren Aymara and Non-aymara of 10-12 Years, Living in High Altitude (3500m) and the Plain (500 m), from Chile. Int. J. Morphol., 27(4):13-8, 2009

Goulding, A.; Taylor, R. W.; Grant, A. M.; Parnell, W. R.; Wilson, N. C. \& Williams, S. M. Waist-to-height ratios in relation to BMI z-scores in three ethnic groups from a representative sample of New Zealand children aged 5-14 years. Int. J. Obes. (Lond.), 34(7):1188-90, 2010.

Hebbelinck, M.; Carter, L. \& De Garay, A. Body buil and somatotype of Olympic swimmers, divers, and water polo players. En: Lewillie, L. \& Clarys, J. P. (Eds.). Swimming II. Baltimore, University Park Press, 1975. pp.285-305.

Hoyo, L. M. \& Sañudo, C. B. Composición corporal y actividad física como parámetros de salud en niños de una población rural de Sevilla. RYCYDE Rev. Int. Cienc. Deporte, 3(6):52-62, 2007.

Kagawa, M.; Byrne, N. M.; King, N. A.; Pal, S. \& Hills, A. P. Ethnic differences in body composition and anthropometric characteristics in Australian Caucasian and urban Indigenous children. Br. J. Nutr., 102(6):938-46, 2009.

Liberona, Y.; Castillo, O.; Engler, V.; Villaroel, L. \& Rozowski, J. Nutritional profile of schoolchildren from different socio-economic levels in Santiago, Chile. Public Health Nutr., 14(1):142-9, 2011.
Lizana Arce, P. J.; Almagià Flores, A. A.; Simpson Levievre, M. C.; Binvignat Gutierrez, O.; Ivanovic Marincovich, D. \& Berral de la Rosa, F. J. Approximation to the secular tendency of the nutritional state and body composition of high school students, V Region, Chile: 19852010. Int. J. Morphol., 29(2):473-4. 2011.

Martínez, C.; Silva, H.; Collipal, E.; Carrasco, V.; Rodríguez, M.; Vargas, R.; Gatica, P. \& Silva, T. Somatotype and nutritional status from 10 to 14 years of age in a sample of Mapuche subjects in the IX Region, Temuco-Chile. Int. J. Morphol., 30(1):241-6, 2012.

Martínez, C.; Reinike, O.; Silva, H.; Carrasco, V.; Collipal, E. \& Jiménez, C. Body composition and nutritional status of 9 to 12 year old municipal schools students sample of Padre las Casas Commune, Araucanía Region, Chile. Int. J. Morphol., 31(2):425-31, 2013.

Marrodán, M. D.; Aréchiga, J. \& Moreno-Romero, S. Cambios somatotípicos Durante el Crecimiento en población Mexicana masculina. Antropo, 1:43-50, 2001. Disponible en: http:// www.didac.ehu.es/antropo/1/1-5/Marrodan.pdf

Ministerio de Salud (MINSAL). Encuesta Nacional de Salud ENS Chile 2009-2010. Santiago de Chile, Ministerio de Salud, Gobierno de Chile, 2010. Disponible en: http://web.minsal.cl/portal/url/item/ bcb03d7bc28b64dfe040010165012d23.pdf

Nightingale, C. M.; Rudnicka, A. R.; Owen, C. G.; Wells, J. C.; Sattar, N.; Cook, D. G. \& Whincup, P. H. Influence of adiposity on insulin resistance and glycemia markers among U.K. Children of South Asian, black African-Caribbean, and white European origin: child heart and health study in England. Diabetes Care, 36(6):1712-9, 2013.

Peña, M. \& Bacallao, J. La obesidad y sus tendencias en la región. Rev. Panam. Salud Pública, 10(2):45-78, 2001.

Silva, H.; Bruneau, J.; Reyno, H. P. \& Bucarey, S. Somatotype and body mass index from a both sexes adolescents' sample in Temuco, Chile. Int. J. Morphol., 21(4):309-13, 2003.

Silva M., H.; Collipal L., E.; Martínez, C. \& Torres, I. Analysis of BMI and somatotype in a sample of adolescents were over weight and obesity in Temuco - Chile. Int. J. Morphol., 26(3):707-11, 2008.

Slaughter, M. H.; Lohman, T. G.; Boileau, R. A.; Horswill, C. A.; Stillman, R. J.; Van Loan, M. D. \& Bemben, D. A. Skinfold equations for estimation of body fatness in children and youth. Hum. Biol., 60(5):709$23,1988$.

\section{Dirección para Correspondencia: José Gastón Bruneau Chávez Universidad Autónoma de Chile Avenida Alemania 01090 \\ Temuco \\ CHILE}

Email: jose.bruneau@uautonoma.cl 